Mathematical Models and Methods in Applied Sciences

Vol. 16, No. 2 (2006) 211-231

(C) World Scientific Publishing Company

\title{
A STABILIZING SUBGRID FOR CONVECTION-DIFFUSION PROBLEM
}

\author{
ALI I. NESLITURK \\ Izmir Institute of Technology, \\ Department of Mathematics, \\ 35430 Izmir, Turkey \\ alinesliturk@iyte.edu.tr \\ Received 27 October 2004 \\ Revised 17 May 2005 \\ Communicated by F. Brezzi
}

\begin{abstract}
A stabilizing subgrid which consists of a single additional node in each triangular element is analyzed by solving the convection-diffusion problem, especially in the case of small diffusion. The choice of the location of the subgrid node is based on minimizing the residual of a local problem inside each element. We study convergence properties of the method under consideration and its connection with previously suggested stabilizing subgrids. We prove that the standard Galerkin finite element solution on augmented grid produces a discrete solution that satisfy the same a priori error estimates that are typically obtained with SUPG and RFB methods. Some numerical experiments that confirm the theoretical findings are also presented.
\end{abstract}

Keywords: The convection-diffusion problem; the stabilized FEM.

AMS Subject Classification: 65N30

\section{Introduction}

It is well known that the exact solution of the convection-diffusion problem may exhibit boundary and internal layers, i.e. very narrow regions where the solution and its derivatives change sharply. If the standard Galerkin Finite Element Method (FEM) with a discretization scale, which is too large to resolve the layers, is employed, the numerical method will produce oscillations that pollute the whole domain. To properly resolve the layers, the mesh size must be of the same size as the ratio between diffusion and convection. However, this choice would make the classical FEM impractical for real-world problems.

Several approaches have been proposed to rectify this problem mostly for convection-dominated cases. Probably the most popular approach where the variational formulation is augmented with the mesh-dependent terms in order to gain control on the derivatives of the solution is known as the Streamline-Upwind Petrov-Galerkin (SUPG) method. ${ }^{9}$ Successful applications of the SUPG method 
together with its many variants to a wide variety of physical problems can be found in the literature. ${ }^{10,14,16}$ The advantage of this approach is not only its generality, but also its error analysis can be performed in many cases of interest. It is well known that the mesh-dependent terms added to the variational formulation corresponds to providing additional diffusion in the streamline direction. The amount of such additional diffusion is tuned by a stabilization parameter $\tau$ that must be chosen properly. Nevertheless, the need for a convincing argument to guide the choice of $\tau$ is still considered as a major drawback of the method.

Another approach to construct a numerical method that produces numerical solutions consistent with the physical configuration of the problem consist in enriching the finite element space by bubble functions. The relationship between the use of bubble functions and the stabilized methods were first studied in Ref. 6 . It turns out that, to find a suitable value for the stabilization parameter, it is crucial to use a special type of bubbles, called Residual-Free Bubbles (RFB), dictated by a local PDE problem related with the original one inside each element. ${ }^{8}$ The formulations using the RFB functions for convection-dominated problems have been analyzed from the theoretical point of view, and a priori error bounds were proved, similar to the ones for the SUPG, for several cases of interest. ${ }^{4,12,13,15,17}$ Since finding the exact solution of the local problem is usually difficult as much as the original one, a suitable strategy to obtain a cheap approximate solution has to be designed. The fact that the local problem is defined over a triangular or quadrilateral domain makes this strategy practical for many problems of interest. Therefore several researchers dealt with the local problem to provide an approximate solution having some level of precision with the use of a suitable subgrid. ${ }^{3,12,7,11}$ However, the resulting numerical methods were lacking a satisfactory error analysis where they reproduce the same behavior as the SUPG or the RFB method. More recently, Brezzi and his coworkers, in Ref. 1, set the abstract conditions on the choice of the subgrid that guarantees the numerical method satisfies the same a prior error estimates as the SUPG and the RFB methods. In Ref. 2, they further constructed a very simple subgrid which consists of a single node that meets the sufficient conditions in Ref. 1 so that the discrete solution on the augmented grid satisfies a priori error bounds.

The method analyzed in this paper emanates from merging concepts of some recent papers of Brezzi and his coworkers. It is based on augmenting a given mesh by adding suitable internal nodes inside each triangle, forming a subgrid by joining the additional points to coarse grid points conveniently and then solving the problem on the augmented grid, that is the original grid plus the subgrid, in the framework of the Standard Galerkin FEM. Here we will choose subgrid consists of just one internal (additional) node plus three vertices within each element. Joining the additional node to the three vertices makes the triangle split into three subgrid triangles. The strategy for choosing the internal node, introduced in Ref. 3 in the context of the pseudo-residual-free bubble method, depends on minimizing the residual of a local problem. The location of the subgrid node dictated by this strategy coincides with the one suggested by Ref. 2 if the element under consideration has one inflow edge. 
We further prove that the method satisfies the abstract conditions in Ref. 1 and so does the same a priori error estimates as the one for the exact RFB method. On the other hand, the internal node added with the subgrid, in practice, can still be eliminated by static condensation, so that the method could still be regarded as a variant of the RFB approach, so-called the pseudo Residual-free Bubble Method. ${ }^{3}$ We will make occasional use of this fact throughout the paper.

The layout of the paper is as follows. In Sec. 2 we briefly recall basic ideas of the SUPG, the RFB and the Standard Galerkin on Augmented Grids. In Sec. 3 we describe our choice of subgrid in one-dimensional problems. In Sec. 4 the choice of subgrid is displayed for two-dimensional problems and some of its properties are studied. The corresponding a prior error estimates for the formulation is also proved in this section. In Sec. 5, we present several numerical experiments to validate the method.

\section{Stabilizing Methods}

Consider the following linear elliptic convection-diffusion problem in a polygonal domain $\Omega$ :

$$
\begin{cases}L u=f & \text { in } \Omega, \\ u=0 & \text { on } \partial \Omega,\end{cases}
$$

where

$$
L u=-\varepsilon \triangle u+\beta \nabla u .
$$

Let $\tau_{h}$ be a regular decomposition of $\Omega$ into triangles $K$, let $h_{K}$ be the diameter of the element $K$ and $h=\max _{K \in \tau_{h}} h_{K}$. We assume that the diffusion coefficient $\varepsilon$ is a positive constant, and that the convection field $\beta$ and the right-hand side $f$ are piecewise constants with respect to the decomposition $\tau_{h}$. Let us recall the classical variational formulation of the problem (2.1): Find $u \in H_{0}^{1}(\Omega)$ such that

$$
a(u, v)=(f, v) \text { for all } v \in H_{0}^{1}(\Omega),
$$

where

$$
a(u, v)=\varepsilon \int_{\Omega} \nabla u \cdot \nabla v d \Omega+\int_{\Omega}(\beta \cdot \nabla u) v d \Omega
$$

is a continuous and coercive bilinear form on the Hilbert space $H_{0}^{1}(\Omega)$, and $(f, v)$ denotes the scalar product of $f$ and $v$ in $L^{2}(\Omega)$. Galerkin approximation of the problem (2.1) consists of taking a finite-dimensional subspace $V_{h}$ of $H_{0}^{1}(\Omega)$, and then solving the variational problem $(2.3)$ in $V_{h}$. Let us consider the finite element space of continuous, piecewise linear elements:

$$
V_{L}=\left\{v \in H_{0}^{1}(\Omega):\left.v\right|_{K} \text { linear for all } K \in \tau_{h}\right\} .
$$

The finite element discretization of the problem (2.3) in $V_{L}$ reads: Find $u_{L} \in V_{L}$ such that

$$
a\left(u_{L}, v_{L}\right)=\left(f, v_{L}\right) \text { for all } v_{L} \in V_{L} .
$$


It is well known that the exact solution of the problem (2.1) will have a boundary layer at the outflow when $\varepsilon \ll \beta h$. In this case, the standard Galerkin finite element approximation (2.6) will typically fail showing strong oscillations near the boundary layer and some stabilization is necessary.

The most classical and extensively used technique to stabilize (2.6) is to use the Streamline-Diffusion Petrov-Galerkin method (SUPG). The SUPG method consist in adding to the bilinear form in (2.6) a mesh-dependent term which introduces a suitable amount of artificial diffusion in the direction of streamlines without upsetting consistency. The amount of such additional diffusion is tuned by a parameter $\tau_{K}$ that must be chosen in a suitable way. In the case of problem (2.1), with linear elements, the SUPG method reads: Find $u_{L} \in V_{L}$ such that

$$
a\left(u_{L}, v_{L}\right)+\sum_{K \in \tau_{h}} \tau_{K} \int_{K}\left(\beta \cdot \nabla u_{L}-f\right)\left(\beta \cdot \nabla v_{L}\right)=\left(f, v_{L}\right) \quad \text { for all } v_{L} \in V_{L},
$$

where $\tau_{K}$ is a stabilization parameter defined element by element depending on the local character of the discretization:

$$
\tau_{K}= \begin{cases}\frac{h_{K}}{2 \beta_{K}} & \text { if } P e_{K} \geq 1, \\ \frac{h_{K}^{2}}{12 \varepsilon} & \text { if } P e_{K}<1,\end{cases}
$$

where $P e_{K}=\beta_{K} h_{K} / 6 \varepsilon$. The scheme (2.7) leads to a numerical solution consistent with the physical configuration of the problem (2.1). Furthermore a priori error estimates for the approximate solution $u_{L}^{S}$ obtained by the SUPG method can be proved to be of type

$$
\begin{gathered}
\varepsilon\left|u-u_{L}^{S}\right|_{H^{1}(\Omega)}^{2}+\sum_{K \in \tau_{h}} h_{K}\left\|\beta \cdot \nabla\left(u-u_{L}^{S}\right)\right\|_{L^{2}(\Omega)}^{2} \\
\leq C \sum_{K \in \tau_{h}}\left(\varepsilon h_{K}^{2 s-2}+h_{K}^{2 s-1}\right)|u|_{H^{s}(\Omega)}^{2},
\end{gathered}
$$

whenever the solution belongs to $H^{s}(\Omega)$ for some $s$ with $1<s \leq 2$. However, the need for a suitable convincing argument to guide the choice of $\tau$ is still considered as a major drawback of the method.

A way to recover the value of $\tau_{K}$ is to use the RFB approach. The idea consists of enlarging the finite element space $V_{L}$. For that, we define, in each $K$, the space of bubbles as $B^{K}=H_{0}^{1}(K)$, the enlarging space $V_{B}$ as $V_{B}=\bigoplus_{K \in \tau_{h}} B^{K}$, and set

$$
V_{h}=V_{L} \bigoplus V_{B}
$$

Thus any $v_{h} \in V_{h}$ can be split into a linear part $v_{L} \in V_{L}$ and a bubble part $v_{B} \in V_{B}$ in a unique way:

$$
v_{h}=v_{L}+v_{B} \in V_{L} \bigoplus V_{B}
$$


and the bubble part itself can be uniquely split element by element:

$$
v_{B}=\sum_{K} v_{B, K}, \quad v_{B, K} \in B_{K} .
$$

The variational problem $(2.3)$ in $V_{h}$ can be written as follows: Find $u_{h}=u_{L}+u_{B} \in$ $V_{L} \oplus V_{B}$, for all $v_{L} \in V_{L}, K \in \tau_{h}$, and $v_{B, K} \in B_{K}$, such that,

$$
\begin{aligned}
a\left(u_{L}+u_{B}, v_{L}\right) & =\left(f, v_{L}\right), \\
a\left(u_{L}+u_{B, K}, v_{B, K}\right)_{K} & =\left(f, v_{B, K}\right)_{K},
\end{aligned}
$$

where $(., .)_{K}$ indicates that the integrals involved are restricted to the element $K$. Solving (2.14) inside each $K$ for $u_{B, K}$ and substituting into the first equation, it can be shown that the effect of the bubbles, which are chosen to be residual-free inside each $K$, can be identified with an additional term that has an identical structure with the mesh-dependent term in the SUPG method. Consequently, the resulting scheme on $V_{L}$ reads: Find $u_{L} \in V_{L}$ such that

$a\left(u_{L}, v_{L}\right)+\sum_{K \in \tau_{h}} \hat{\tau}_{K} \int_{K}\left(\beta \cdot \nabla u_{L}-f\right)\left(\beta \cdot \nabla v_{L}\right)=\left(f, v_{L}\right) \quad$ for all $v_{L} \in V_{L}$,

where

$$
\hat{\tau}_{K}=\frac{1}{|K|} \int_{K} b_{K} d K
$$

and $b_{K}$ solves the following boundary value problem in $K$ :

$$
\begin{cases}L b_{K}=-\varepsilon \Delta b_{K}+\beta_{K} \cdot \nabla b_{K}=1 & \text { in } K \\ b_{K}=0 & \text { on } \partial K\end{cases}
$$

A priori error estimates for the RFB method were proved for linear elements in Ref. 4: If the solution belongs to $H^{s}(\Omega)$ for some $s$ with $1<s \leq 2$, then

$$
\begin{gathered}
\varepsilon\left|u-u_{L}^{R}\right|_{H^{1}(\Omega)}^{2}+\sum_{K \in \tau_{h}} h_{K}\left\|\beta \cdot \nabla\left(u-u_{L}^{R}\right)\right\|_{L^{2}(\Omega)}^{2} \\
\leq C \sum_{K \in \tau_{h}}\left(\varepsilon h_{K}^{2 s-2}+h_{K}^{2 s-1}\right)|u|_{H^{s}(\Omega)}^{2}
\end{gathered}
$$

where $u_{L}^{R}$ is the linear component of the RFB solution. See Refs. 5 and 12 for additional results.

However, the method (2.15) depends on the solution of the local problem (2.17) whose solution may be difficult as much as the original problem. Therefore several numerical methods were proposed to compute an approximate solution of the problem (2.17). ${ }^{3,2,12,7}$ The common point of these attempts was to construct a low-dimensional subspace $B_{h}^{K} \subset B^{K}$ in such a way that the solution of the discrete local problem,

$$
\text { Find } b_{h}^{K} \in B_{h}^{K} \quad \text { such that } a\left(b_{h}^{K}, b_{h}\right)=\left(1, b_{h}\right) \quad \forall b_{h} \in B_{h}^{K} \text {, }
$$


could produce a solution $b_{h}^{K}$ such that

$$
\int_{K} b_{h}^{K} \simeq \int_{K} b_{K}
$$

From a different point of view, these methods can be regarded as a standard Galerkin method on an augmented grid for which the finite element space $V_{h}^{A}$ is formed by the original space $V_{L}$ augmented with the subgrid space $B_{h}^{K}$; i.e.

$$
V_{h}^{A}=V_{L} \bigoplus_{K \in \tau_{h}} B_{h}^{K} .
$$

It can be proved that if $B_{h}^{K}$ satisfies certain sufficient conditions, then the solution of the problem

$$
\left\{\begin{array}{l}
\text { Find } u_{h} \in V_{h}^{A} \text { such that } \\
a\left(u_{h}, v\right)=(f, v) \quad \forall v \in V_{h}^{A}
\end{array}\right.
$$

satisfies the same type a priori error bounds as SUPG and RFB methods. ${ }^{1}$ In the following sections we are going to display how to choose a convenient subgrid consisting of a single additional node per element, use some results from Ref. 1 to show that the present choice of subgrid satisfies the sufficient conditions and thus the same error estimates, as in the SUPG and RFB methods, hold true for the corresponding numerical method. Before we present the method and analyze its properties, we study the one-dimensional case, although it is not our main interest, just to have a better understanding of the behavior of the numerical method under investigation.

\section{The Choice of Subgrid in $1 \mathrm{D}$}

Let us consider the problem (2.1) in a one-dimensional domain, scaled to the unit interval $\mathrm{I}=(0,1)$ for the sake of simplicity:

$$
\left\{\begin{array}{l}
-\varepsilon u^{\prime \prime}+\beta u^{\prime}=f \quad \text { in }(0,1), \\
u(0)=u(1)=0
\end{array}\right.
$$

Let $\tau_{h}$ be a discretization of $I$ into $N$ subintervals $K_{j}=\left(x_{j-1}, x_{j}\right), j=1, \ldots, N$ with $0=x_{0}<x_{1}<\cdots<x_{N}=1$. We shall also assume that the decomposition is uniform $\left(h=1 / N=x_{j}-x_{j-1}\right)$. However, all the discussions will take place at the element level, therefore it will be valid for a general decomposition. Let us further assume that the diffusion parameter $\varepsilon$ is a positive constant and that the convection field $\beta$ and the source function $f$ are piecewise constants with respect to the decomposition $\tau_{h}$. The residual-free bubble formulation of the problem can be directly obtained from $(2.15)$ by restating $V_{L}$ concerning the decomposition $\tau_{h}$ over $I$. The resulting numerical method then reads: Find $u_{L} \in V_{L}$ such that

$\varepsilon\left(u_{L}^{\prime}, v_{L}^{\prime}\right)+\left(\beta u_{L}^{\prime}, v_{L}\right)+\sum_{j=1}^{N} \hat{\tau}_{K_{j}} \int_{x_{j-1}}^{x_{j}}\left(\beta u_{L}^{\prime}-f\right)\left(\beta v_{L}^{\prime}\right)=\left(f, v_{L}\right) \quad$ for all $v_{L} \in V_{L}$, 
where

$$
\hat{\tau}_{K_{j}}=\frac{1}{h} \int_{x_{j-1}}^{x_{j}} b_{K_{j}} d x .
$$

We shall mostly drop the index $j$ in the notation as the following discussion takes place in a typical element $K_{j}$. The residual-free bubble function $b_{K}$ in the definition of $\hat{\tau}_{K}$ solves the following boundary value problem defined in $K$ :

$$
\left\{\begin{array}{l}
-\varepsilon b_{K}^{\prime \prime}+\beta_{K} b_{K}^{\prime}=1 \quad \text { in }\left(x_{j-1}, x_{j}\right), \\
b_{K}\left(x_{j-1}\right)=b_{K}\left(x_{j}\right)=0 .
\end{array}\right.
$$

Let us find an approximation to $b_{K}$ over a subgrid inside $K$ that contains just one additional node $P=P_{K}$. We will use $x_{P}$ instead of $P$ whenever it is appropriate. Suppose $\beta>0$. The case $\beta<0$ can be treated similarly just by exchanging the roles of $x_{j-1}$ and $x_{j}$. We construct a continuous and piecewise linear function $b_{P}$ in $K$ with the property that $b_{P}$ has value one at $x_{P}$ and value zero at the other points of the subgrid (see Fig. 1). Let $b_{P}^{*}(x)=\alpha b_{P}(x)$ be the classical Galerkin solution of (3.4) over subgrid in $K$ :

$$
\varepsilon\left(b_{P}^{*}, b_{P}\right)_{K}+\left(\beta b_{P}^{*}, b_{P}\right)_{K}=\left(1, b_{P}\right)_{K} \quad \forall b_{P} .
$$

This equation determines $\alpha$ in terms of $x_{P}$. The main guideline for the choice of $x_{P}$ will be to minimize $L_{1}$ norm of the residual of the bubble equation inside a fixed element $K$. This amounts to choosing $x_{P}$ such that

$$
J(P)=\int_{x_{j-1}}^{x_{j}}\left|-\varepsilon b_{P}^{* \prime \prime}+\beta b_{P}^{* \prime}-1\right| d x
$$

is minimum. The integral above has to be considered in the sense of distributions. The enforcement of the condition (3.6) reveals that

$$
x_{P} \geq x_{j}-\frac{2 \varepsilon}{\beta} \quad \text { with } x_{P} \in\left(x_{j-1}, x_{j}\right) .
$$

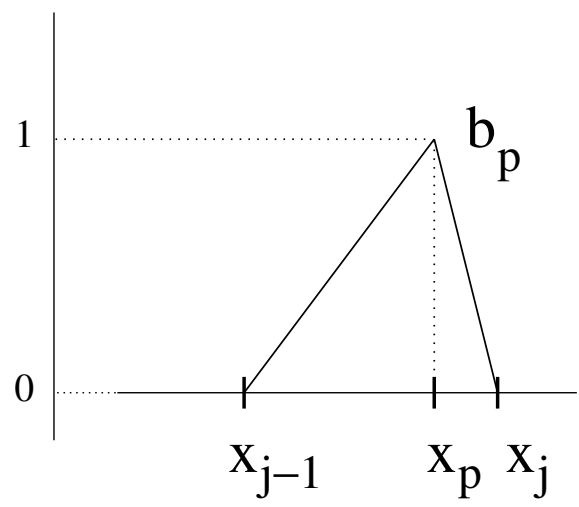

Fig. 1. Definition of the function $b_{P}$. 


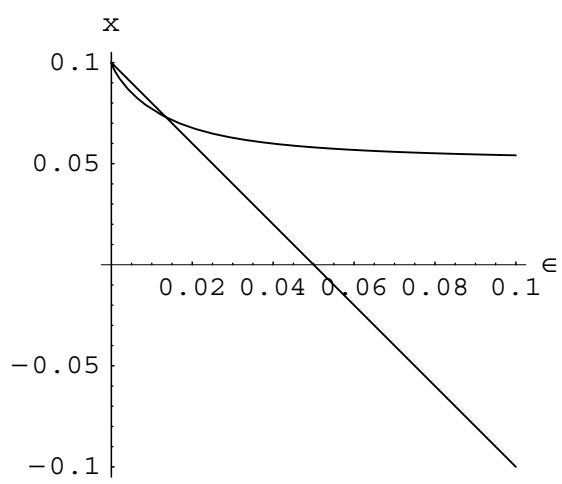

Fig. 2. Comparison of $x_{\max }$ (curved) with $x_{P}$ (line) with respect to $\varepsilon: N=10, \beta=1$.

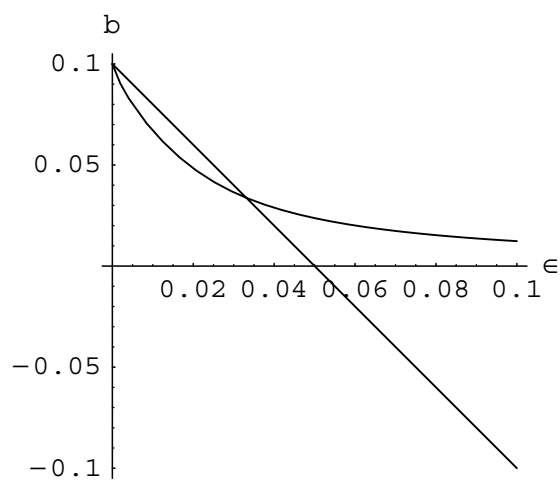

Fig. 3. Comparison of $b_{K}\left(x_{\max }\right)$ (curved) with $b_{P}^{*}\left(x_{P}\right)$ (line) with respect to $\varepsilon: N=10, \beta=1$.

We leave the details of this computation to the next section as they are just a restriction of the process displayed for two-dimensional case. Now, although any point in the interval $S=\left(x_{j}-\frac{2 \varepsilon}{\beta}, x_{j}\right)$ minimizes the integral in (3.6), we take $x_{P}$ to be the leftmost point, i.e. $x_{P}=x_{j}-\frac{2 \varepsilon}{\beta}$ because it provides the biggest stabilizing effect among the points of $K$ compatible with the constraint $x_{P} \in S$. On the other hand, a comparison of $b_{P}^{*}$ with the exact solution $b_{K}$ in (3.4) shows that the description (3.7) for $x_{P}$ is good only if $\varepsilon$ is small enough. (see Figs. 2 and $3: x_{\max }$ is the point where the exact solution $b_{K}$ in (3.4) has its maximum; $b_{K}\left(x_{\max }\right)$ is the value of $b_{K}$ at $x_{\max } ; b_{P}^{*}\left(x_{P}\right)$ is defined accordingly). Thus, to obtain a reasonable approximation through the numerical method (2.22), it is appropriate to define the point $x_{P}$, with a smooth transition, as follows:

$$
x_{P}= \begin{cases}x_{j}-\frac{2 \varepsilon}{\beta} & \text { if } \varepsilon \leq \frac{h \beta}{6}, \\ x_{j}-\frac{h}{3} & \text { if } \varepsilon>\frac{h \beta}{6} .\end{cases}
$$



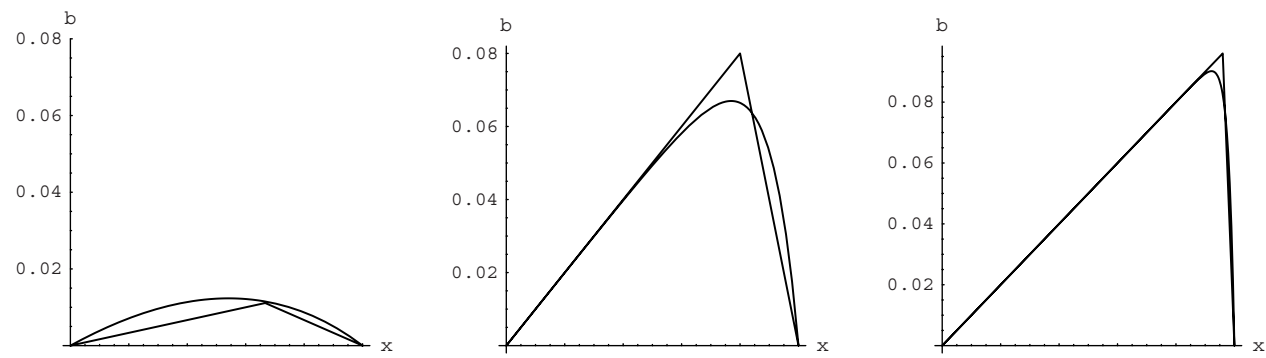

Fig. 4. Comparison of $b_{K}$ (curved) with the approximate solution $b_{P}^{*}$ (line) in a typical element $K$ for $\varepsilon=0.1,0.01,0.002$, respectively $\left(N=10, \beta=1, x_{P}\right.$ is defined as in (3.8)).

We note that the location of the subgrid node $x_{P}$ is the same as the location of subgrid suggested by the method presented in Ref. 7. Therefore, both methods produce the same numerical results.

On the other hand, by eliminating the bubbles' degrees of freedom, it is possible to write the numerical method as a finite difference scheme on the coarse mesh when $\beta$ and $f$ are constants throughout the domain $I$ : Define $u_{j}=u_{L}\left(x_{j}\right)$, use the method (3.2) and expand the integrals to obtain

$$
\begin{cases}\frac{\beta}{h}\left(u_{j}-u_{j-1}\right)=f_{j} & \text { if } \varepsilon \leq \frac{h \beta}{6}, \\ -\left(\varepsilon+\frac{\beta^{2} h^{2}}{18 \varepsilon}\right) \frac{u_{j+1}-2 u_{j}+u_{j-1}}{h^{2}}+\beta \frac{u_{j+1}-u_{j-1}}{2 h}=f_{j} & \text { if } \varepsilon>\frac{h \beta}{6} .\end{cases}
$$

From (3.9), it is appropriate to remark two points here: (a) In Ref. 7, the location of subgrid node is chosen such that the contribution of the downwind node to the subgrid node annihilates in each element. This is consistent with the result in (3.9) as the numerical method is just a backward difference approximation of the convection term. (b) When $\varepsilon$ is large enough, the method corresponds to the wellknown classical artificial diffusion method with an additional diffusion $\beta^{2} h^{2} / 18 \varepsilon$. The amount of diffusion can be tuned by changing the transition value of $\varepsilon$ in (3.8).

Now we return to our main thread and display how this strategy can be applied in two-dimensional problems. We further study the properties of the method under consideration and its connection with the subgrid suggested in Ref. 2.

\section{The Choice of Subgrid in $2 \mathrm{D}$}

Let us consider a subgrid that contains just one additional node $P=P_{K}$ in each triangular element. The node $P$ is joined to the three vertices splitting the triangle $K$ into three sub-triangles. We will choose the point $P$ along one of the three medians of $K$. The choice of the median and the location of $P$, which may differ from element to element, will be made precise in the sequel. Take an element $K$ and a local numbering for its vertices $V_{i}(i=1,2,3)$ using the counterclockwise order. 
Let us denote basis functions on $K$ by $b_{p}, \varphi_{1}, \varphi_{2}, \varphi_{3}$ where

$$
\begin{array}{lll}
b_{P}(P)=1, & b_{P}\left(V_{i}\right)=0 & i=1,2,3, \\
\varphi_{i}\left(V_{i}\right)=1, & \varphi_{i}\left(V_{j}\right)=0 & j \neq i,
\end{array}
$$

Thus the basis function attached to the point $P$ has support contained in $K$ and the other three basis functions have value one at one vertex and zero at $P$ and other vertices. We further denote the edges of $K$ by $\mathbf{e}_{i}$ opposite to $V_{i}$, the length of $\mathbf{e}_{i}$ by $\left|\mathbf{e}_{i}\right|$, the outward unit normal to $\mathbf{e}_{i}$ by $\mathbf{n}_{i}$ and $\nu_{i}=\left|\mathbf{e}_{i}\right| \mathbf{n}_{i}$. We note that the actual numbering of vertices $V_{i}$ will be chosen according to the direction of $\beta$. In order to choose the position of $P$, we have to distinguish among two cases:

\subsection{Case 1: Two inflow edges}

Let the inflow boundary make up of two edges and let $\mathbf{e}_{2}$ and $\mathbf{e}_{3}$ be two inflow edges (Fig. 5). The position of $P$ along the median from $V_{1}$ will be determined by the following condition: Choose $P$ such that the value of the integral

$$
J(P)=\int_{K}\left|-\varepsilon \triangle b_{P}^{*}+\beta \cdot \nabla b_{P}^{*}-1\right| d K
$$

is minimum where $b_{P}^{*}(\mathbf{x})=\alpha(P) b_{P}(\mathbf{x})$ is the unique solution of

$$
a_{K}\left(b_{P}^{*}, b_{P}\right)=\left(1, b_{P}\right) \quad \forall b_{P} .
$$

We remark again that the integral in (4.2) has to be intended in the sense of measures. Using the integration by parts and the fact that $\beta$ is constant in each element, observe that

$$
\alpha(P)=\frac{\int_{K} b_{P} d K}{\varepsilon \int_{K}\left|\nabla b_{P}\right|^{2} d K} .
$$

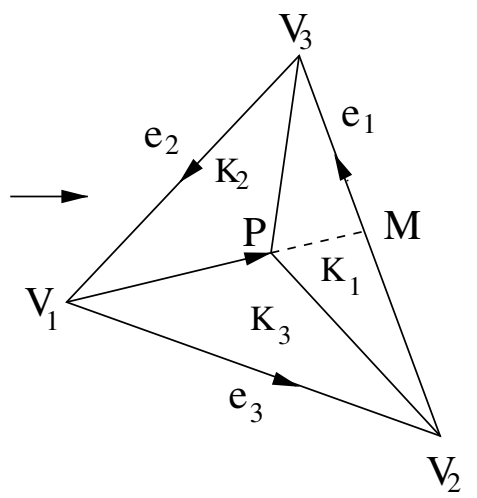

(a)

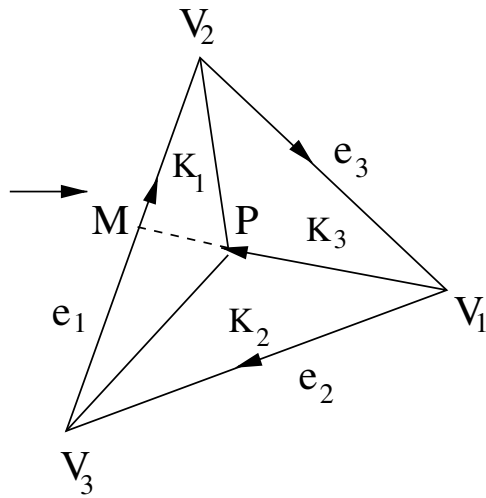

(b)

Fig. 5. Types of inflow boundary: (a) two inflow edges, (b) one inflow edge. 
The criteria (4.2) were also used by Brezzi and his coworkers in Ref. 3 in the context of the pseudo Residual-free Bubbles. According to Ref. 3, it is possible to write the integral $J(P)$ as follows:

$$
J(P)=\frac{2}{3}|K|+\int_{K}\left|\beta \cdot \nabla b_{P}^{*}-1\right| d K .
$$

We note that the first term on the right-hand side of (4.5) is independent of $P$. Let $g_{i}=\left.\left(\beta \cdot \nabla b_{P}^{*}-1\right)\right|_{K_{i}}$. Note that $g_{i}$ is constant over the corresponding sub-triangle $K_{i}$ and that $g_{1}$ is negative over $K_{1}$. Since

$$
\sum_{i=1,2,3} \int_{K_{i}} g_{i}=-|K| \text { and } \sum_{i=1,2,3} \int_{K_{i}}\left|g_{i}\right| \geq|K|,
$$

$J(P)$ attains its minimum if both $g_{2}$ and $g_{3}$ are negative; that is

$$
\left.\left(\beta \cdot \nabla b_{P}^{*}\right)\right|_{K_{2}} \leq 1 \text { and }\left.\left(\beta \cdot \nabla b_{P}^{*}\right)\right|_{K_{3}} \leq 1
$$

The set of points on the median $V_{1} M$ can be described as a function depending on a single parameter $t: P=(1-t) V_{1}+t M$ where $0<t<1$. Then, using Eq. (4.4), $\nabla b_{P}=-\left|\mathbf{e}_{i}\right| \mathbf{n}_{i} / 2\left|K_{i}\right|,\left|K_{1}\right|=(1-t)|K|,\left|K_{2}\right|=t|K| / 2$ and $\left|K_{3}\right|=t|K| / 2$, the conditions in (4.6) imply that the parameter $t$ must satisfy two inequalities below simultaneously;

$$
\begin{aligned}
& -\frac{2|K|}{3 \varepsilon}\left(\beta, \nu_{2}\right) \leq \frac{t}{2}\left(\frac{\left|\mathbf{e}_{1}\right|^{2}}{1-t}+\frac{2\left|\mathbf{e}_{2}\right|^{2}}{t}+\frac{2\left|\mathbf{e}_{3}\right|^{2}}{t}\right), \\
& -\frac{2|K|}{3 \varepsilon}\left(\beta, \nu_{3}\right) \leq \frac{t}{2}\left(\frac{\left|\mathbf{e}_{1}\right|^{2}}{1-t}+\frac{2\left|\mathbf{e}_{2}\right|^{2}}{t}+\frac{2\left|\mathbf{e}_{3}\right|^{2}}{t}\right) .
\end{aligned}
$$

Without loss of generality, assume

$$
\left(\beta, \nu_{3}\right) \geq\left(\beta, \nu_{2}\right)
$$

thus the values of $t$ that solve the inequality (4.7) will also satisfy the inequality (4.8) automatically. Recall that, for any triangle with sides $e_{1}, e_{2}$ and $e_{3}$, we have the identity

$$
2\left(\left|\mathbf{e}_{2}\right|^{2}+\left|\mathbf{e}_{3}\right|^{2}\right)=\left|\mathbf{e}_{1}\right|^{2}+\left|\mathbf{e}_{2}-\mathbf{e}_{3}\right|^{2} .
$$

Let us rewrite (4.7) by using the identity (4.10);

$$
\begin{aligned}
& -\frac{2|K|}{3 \varepsilon}\left(\beta, \nu_{2}\right) \leq \frac{t}{1-t} \frac{\left|\mathbf{e}_{1}\right|^{2}}{2}+\left|\mathbf{e}_{2}\right|^{2}+\left|\mathbf{e}_{3}\right|^{2}, \\
& -\frac{2|K|}{3 \varepsilon}\left(\beta, \nu_{2}\right) \leq \frac{t}{1-t} \frac{\left|\mathbf{e}_{1}\right|^{2}}{2}+\frac{\left|\mathbf{e}_{1}\right|^{2}}{2}+\frac{\left|\mathbf{e}_{2}-\mathbf{e}_{3}\right|^{2}}{2} .
\end{aligned}
$$

Solving the last inequality for $t$ we get

$$
1>t \geq t_{1}^{*}=1+\frac{\varepsilon\left|\mathbf{e}_{1}\right|^{2}}{\varepsilon\left|\mathbf{e}_{2}-\mathbf{e}_{3}\right|^{2}+\frac{4}{3}|K|\left(\beta, \nu_{2}\right)} .
$$

As any value of $t$ in the interval $\left(t_{1}^{*}, 1\right)$ minimizes the integral $J(P)$, we choose $t=t_{1}^{*}$, the one that gives the farthest point from $M$. Because, in addition to 
making sub-triangles $K_{i}, i=1,2,3$ more regular, it usually provides the biggest stabilizing effect among the points in the interval $\left(t_{1}^{*}, 1\right)$ (see Remark 4.4).

On the other hand, for diffusion dominated problems, this type of stabilization would be unnecessary, and actually the value of $t$ given by (4.13) becomes meaningless (see $1 \mathrm{D}$ case). Therefore as the actual value for $t$, we take

$$
\begin{cases}t=t_{1}^{*} & \text { if } \varepsilon \leq \varepsilon_{1}^{*}=\frac{-4|K|\left(\beta, \nu_{2}\right) / 3}{3\left|\mathbf{e}_{1}\right|^{2}+\left|\mathbf{e}_{2}-\mathbf{e}_{3}\right|^{2}}, \\ t=2 / 3 & \text { otherwise. }\end{cases}
$$

Note that the choice of $\varepsilon_{1}^{*}$ gives a continuous dependence of $t$ upon $\varepsilon$.

Remark 1. If one of the inflow edges is parallel to the flow field $\beta$, say $\mathbf{e}_{3} / / \beta$, then $\left.\beta \cdot \nabla b_{P}^{*}\right|_{K_{3}}=0$ and $g_{3}$ becomes negative on sub-triangle $K_{3}$. In this case, the second inequality in (4.6) is satisfied automatically and it remains to solve the inequality $\left.\left(\beta \cdot \nabla b_{P}^{*}\right)\right|_{K_{2}} \leq 1$ which, in explicit form, is given by (4.7). Following the same steps, the interval in which the values of $t$ minimize the integral $J(P)$ is obtained as

$$
1>t \geq t_{1}^{*}=1+\frac{\varepsilon\left|\mathbf{e}_{1}\right|^{2}}{\varepsilon\left|\mathbf{e}_{2}-\mathbf{e}_{3}\right|^{2}+\frac{4}{3}|K|\left(\beta, \nu_{2}\right)} .
$$

Remark 2. Another effective way of choosing the position of $P$ was suggested and analyzed by Brezzi et al. in Ref. 2: Choose $P$ such that

$$
a_{K}\left(\varphi_{2}, b_{P}\right)+a_{K}\left(\varphi_{3}, b_{P}\right)=0
$$

which determines $t$

$$
t_{1}^{* *}=1+\frac{\varepsilon\left|\mathbf{e}_{1}\right|^{2}}{\varepsilon\left|\mathbf{e}_{2}-\mathbf{e}_{3}\right|^{2}-\frac{2}{3}|K|\left(\beta, \nu_{1}\right)} .
$$

Using the relation $\nu_{1}+\nu_{2}+\nu_{3}=0$, we have

$$
-\frac{2}{3}|K|\left(\beta, \nu_{1}\right)=\frac{2}{3}|K|\left(\beta, \nu_{2}\right)+\frac{2}{3}|K|\left(\beta, \nu_{3}\right) \geq \frac{4}{3}|K|\left(\beta, \nu_{2}\right)
$$

due to the assumption (4.9), which leads us to $t_{1}^{*} \geq t_{1}^{* *}$. Equality holds when $\left(\beta, \nu_{2}\right)=\left(\beta, \nu_{3}\right)$ and both criteria (4.2) and (4.15) produces the same result for the location of $P$.

\subsection{Case 2: One inflow edge}

Let the inflow boundary make up of one edge and let $\mathbf{e}_{1}$ be the inflow edge (Fig. 5). In this case we use again the condition (4.2) to determine the location of $P$. Since $g_{2}$ and $g_{3}$ are already negative in this case, $J(P)$ becomes minimum if $g_{1}$ is negative. That is,

$$
\left.\left(\beta \cdot \nabla b_{P}^{*}\right)\right|_{K_{1}} \leq 1
$$

With the same notation as in Case 1 , that leads us to the inequality

$$
\begin{aligned}
-\frac{2|K|}{3 \varepsilon}\left(\beta, \nu_{1}\right) & \leq(1-t)\left(\frac{\left|\mathbf{e}_{1}\right|^{2}}{1-t}+\frac{2\left|\mathbf{e}_{2}\right|^{2}}{t}+\frac{2\left|\mathbf{e}_{3}\right|^{2}}{t}\right) \\
& =\frac{1}{t}\left(2\left|\mathbf{e}_{2}\right|^{2}+2\left|\mathbf{e}_{2}\right|^{2}\right)-\left|\mathbf{e}_{2}-\mathbf{e}_{3}\right|^{2},
\end{aligned}
$$


where we have used the identity (4.10). Solving the inequality (4.19) for $t$, we get

$$
0<t \leq t_{2}^{*}=\frac{\varepsilon\left(\left|\mathbf{e}_{2}\right|^{2}+\left|\mathbf{e}_{3}\right|^{2}\right)}{\varepsilon\left|\mathbf{e}_{2}-\mathbf{e}_{3}\right|^{2} / 2-|K|\left(\beta, \nu_{1}\right) / 3} .
$$

Again we will use this result only in convection-dominated cases. In particular we take

$$
\begin{cases}t=t_{2}^{*} \quad \text { if } \varepsilon \leq \varepsilon_{2}^{*}=\frac{-2|K|\left(\beta, \nu_{1}\right) / 3}{\left|\mathbf{e}_{1}\right|^{2}+4\left|\mathbf{e}_{2}\right|^{2}+4\left|\mathbf{e}_{3}\right|^{2}} \\ t=1 / 3 \quad \text { otherwise. }\end{cases}
$$

Notice that $t=1 / 3$ when $\varepsilon=\varepsilon_{2}^{*}$ thus the value of $t$ depends on $\varepsilon$ continuously. Since we have $0<t \leq 1 / 3$ for $0<\varepsilon \leq \varepsilon_{2}^{*}$, it is obvious that

$$
0<t \leq \frac{1}{3} \quad \forall \varepsilon>0 .
$$

Remark 3. In Ref. 2, Brezzi and his coworkers use the criteria

$$
a_{K}\left(\varphi_{1}, b_{P}\right)=0
$$

to determine the location of $P$ in Case 2. This gives

$$
t_{2}^{* *}=\frac{\varepsilon\left(\left|\mathbf{e}_{2}\right|^{2}+\left|\mathbf{e}_{3}\right|^{2}\right)}{\varepsilon\left|\mathbf{e}_{2}-\mathbf{e}_{3}\right|^{2} / 2-|K|\left(\beta, \nu_{1}\right) / 3}
$$

which is exactly equal to $t_{2}^{*}$.

Remark 4. It is also possible to choose the location of the subgrid node $P$ so that the value of the stability parameter is maximum. Although this choice is not necessarily stabilizing the numerical method, it is interesting to study some of its properties. Recall that $b_{P}^{*}(\mathbf{x})=\alpha(P) b_{P}(\mathbf{x})$ and the corresponding stabilization parameter $\tilde{\tau}_{K}$ is given by

$$
\tilde{\tau}_{K}=\frac{1}{|K|} \int_{K} b_{P}^{*}
$$

Since the stabilization parameter $\tilde{\tau}_{K}$ is a function of a single parameter along the line segment $V_{1} M$, we can find its maximum easily. Along the median $V_{1} M$, we have $\tilde{\tau}_{K}$ and its derivative as follows:

$$
\begin{aligned}
\tilde{\tau}_{K} & =\frac{4|K| / 9 \varepsilon}{\frac{\left|\mathbf{e}_{1}\right|^{2}}{1-t}+\frac{2\left|\mathbf{e}_{2}\right|^{2}}{t}+\frac{2\left|\mathbf{e}_{3}\right|^{2}}{t}}, \\
\frac{d \tilde{\tau}_{K}}{d t} & =\frac{4|K|}{9 \varepsilon} \frac{\left(2\left|\mathbf{e}_{2}\right|^{2}+2\left|\mathbf{e}_{3}\right|^{2}\right)(1-t)^{2}-\left|\mathbf{e}_{1}\right|^{2} t^{2}}{\left(2\left|\mathbf{e}_{2}\right|^{2}+2\left|\mathbf{e}_{3}\right|^{2}\right)(1-t)^{2}+\left|\mathbf{e}_{1}\right|^{2} t^{2}} .
\end{aligned}
$$

It can be shown that, by using (4.10), the value of $t$ that makes the denominator zero in (4.27) is given by

$$
t=\frac{\left|\mathbf{e}_{2}-\mathbf{e}_{3}\right|^{2}+\left|\mathbf{e}_{1}\right|^{2}}{\left|\mathbf{e}_{2}-\mathbf{e}_{3}\right|^{2}}>1
$$

which is not in the interval $(0,1)$. Thus, to find the critical values of $t$, we only need to find roots of numerator in (4.27). This gives a quadratic equation in $t$ whose 
roots are given by

$$
t^{-,+}=\frac{2 A^{2} \mp \sqrt{2} A\left|\mathbf{e}_{1}\right|}{2 A^{2}-\left|\mathbf{e}_{1}\right|^{2}}, \quad \text { where } A=\sqrt{\left|\mathbf{e}_{2}\right|^{2}+\left|\mathbf{e}_{3}\right|^{2}} .
$$

Observe, from (4.10), $\sqrt{2} A \geq\left|\mathbf{e}_{1}\right|$ and that

$$
t^{+}=\frac{2 A^{2}+\sqrt{2} A\left|\mathbf{e}_{1}\right|}{2 A^{2}-\left|\mathbf{e}_{1}\right|^{2}} \geq \frac{2 A^{2}+\left|\mathbf{e}_{1}\right|^{2}}{2 A^{2}-\left|\mathbf{e}_{1}\right|^{2}}>1 .
$$

Thus $t^{-}$is the only critical point in $(0,1)$. Indeed

$$
t^{-}=\frac{2 A^{2}-\sqrt{2} A\left|\mathbf{e}_{1}\right|}{2 A^{2}-\left|\mathbf{e}_{1}\right|^{2}} \leq \frac{2 A^{2}-\left|\mathbf{e}_{1}\right|^{2}}{2 A^{2}-\left|\mathbf{e}_{1}\right|^{2}}=1
$$

since $\sqrt{2} A \geq\left|\mathbf{e}_{1}\right|$. On the other hand, a calculation shows that $t^{-}>1 / 2$ :

$$
\begin{aligned}
t^{-} & =\frac{2 A^{2}-\sqrt{2} A\left|\mathbf{e}_{1}\right|}{2 A^{2}-\left|\mathbf{e}_{1}\right|^{2}} \cdot \frac{2 A^{2}+\sqrt{2} A\left|\mathbf{e}_{1}\right|}{2 A^{2}+\sqrt{2} A\left|\mathbf{e}_{1}\right|} \\
& =\frac{2 A^{2}\left(2 A^{2}-\left|\mathbf{e}_{1}\right|^{2}\right)}{\left(2 A^{2}-\left|\mathbf{e}_{1}\right|^{2}\right)\left(2 A^{2}+\sqrt{2} A\left|\mathbf{e}_{1}\right|\right)} \\
& =\frac{2 A}{2 A+\sqrt{2}\left|\mathbf{e}_{1}\right|}>\frac{2 A}{2 A+2 A}=\frac{1}{2}
\end{aligned}
$$

since $2 A>\sqrt{2}\left|\mathbf{e}_{1}\right|$.

In brief, the point $P$ corresponding to the parameter $t^{-}$is independent of $\varepsilon$ and it may not stabilize the method. The number $t^{-}$can be bigger or smaller than $t^{*}$ as it depends only on the lengths of sides of the triangle. Furthermore $t^{-}$stays in the interval $\left(\frac{1}{2}, 1\right)$. If the lengths of the sides are equal to each other, then the value of $t^{-}$is $2 / 3$.

Now we return to the error analysis of the method (2.22) with the present choice of the subgrid. In Ref. 1, Brezzi and Marini set abstract assumptions on a subgrid in order to keep the same performance for the corresponding method as we have for the exact RFB Method. We restate these results from Ref. 2 as a particular case of more general results in Ref. 1. Before stating the theorem, we need to introduce two functions; $\varphi$ and $v_{L}(\varphi)$ which have the following descriptions: To every $\varphi$, we associate the function $v_{L}(\varphi)$ defined as the unique function of the form $v_{L}(\varphi)=\varphi+\mu b_{P}$ that satisfies $a_{K}\left(v_{L}(\varphi), b_{P}\right)=0$.

Theorem 4.1. Assume that, in each element $K$, the subgrid is made of a single internal node $P=P(K)$, and let $b_{P}$ be the bubble defined in (4.1). Assume further that the bubble space satisfies the following two assumptions

$$
\exists C_{1}: \forall K \in \tau_{h}, \quad\left\|b_{P}\right\|_{0, K} \leq C_{1} h_{K}^{1 / 2} \varepsilon^{1 / 2}\left|b_{P}\right|_{1, K}
$$

and

$$
\exists C_{2}: \forall K \in \tau_{h}, \forall \varphi \in P_{1}, \quad\|\beta \cdot \nabla \varphi\|_{0, K} \leq C_{2} h_{K}^{-1 / 2} \varepsilon^{1 / 2}\left\|\nabla v_{L}(\varphi)\right\|_{0, K} .
$$

Let $u$ and $u_{h}$ be the solutions of (2.3) and (2.22) respectively, and assume that $u \in H^{s}(\Omega)$ for some $s$ with $1<s \leq 2$. Then there exists a constant $C$, independent 
of $h$, such that

$\varepsilon\left|u-u_{h}\right|_{H^{1}(\Omega)}^{2}+\sum_{K \in \tau_{h}} h_{K}\left\|\beta \cdot \nabla\left(u-u_{h}\right)\right\|_{L^{2}(\Omega)}^{2} \leq C \sum_{K \in \tau_{h}}\left(\varepsilon h_{K}^{2 s-2}+h_{K}^{2 s-1}\right)|u|_{H^{s}(\Omega)}^{2}$.

We will prove two lemmas that give us estimates on the values of $b_{P}^{*}$ at the point $P$ in Case 1 and Case 2. These results will imply that the conditions (4.33) and (4.34) in Theorem 4.1 are satisfied for our present choice of $P$. Thus we will have, by Theorem 4.1, that the numerical method (2.22) with our choice of the subgrid produces a discrete solution that satisfies a priori error estimate (4.35) with a constant independent of $h$.

Lemma 4.1. Assume that, in Case $1, \varepsilon \leq \varepsilon_{1}^{*}$. Then the function $b_{P}^{*}$, the solution of (4.3), satisfies the inequality

$$
C_{*, 1} h_{K} \leq b_{P}^{*}(P) \leq C_{1}^{*} h_{K}
$$

where $C_{1}^{*}$ and $C_{*, 1}$ depend only on $\beta$ and on the minimum angle in $K$.

Proof. Let us recall that

$$
b_{P}^{*}(\mathbf{x})=\alpha(P) b_{P}(\mathbf{x}) \quad \text { where } \alpha(P)=\frac{\int_{K} b_{P} d K}{\varepsilon \int_{K}\left|\nabla b_{P}\right|^{2} d K} .
$$

Since $b_{P}(P)=1$ by definition, it remains to estimate $\alpha(P)$. Observe that

$$
\int_{K}\left|\nabla b_{P}\right|^{2} d K=\sum_{j} \int_{K_{j}}\left|\nabla b_{P}\right|^{2} d K=\sum_{j} \int_{K_{j}} \frac{\left|\mathbf{e}_{j}\right|^{2}}{4\left|K_{j}\right|^{2}} d K=\sum_{j} \frac{\left|\mathbf{e}_{j}\right|^{2}}{4\left|K_{j}\right|}
$$

which enables us to write $\alpha(P)$ in terms of the parameter $t_{1}^{*}$. Using the representation of $\left|K_{j}\right|$ in terms of $t_{1}^{*}$, and the identity (4.10), we get

$$
\begin{aligned}
\alpha(P) & =\frac{4|K|^{2}}{3} \frac{1}{\varepsilon\left(\frac{\left|\mathbf{e}_{1}\right|^{2}}{1-t_{1}^{*}}+\frac{2\left|\mathbf{e}_{2}\right|^{2}}{t_{1}^{*}}+\frac{2\left|\mathbf{e}_{3}\right|^{2}}{t_{1}^{*}}\right)} \\
& =\frac{4|K|^{2}}{3} \frac{t_{1}^{*}\left(1-t_{1}^{*}\right)}{\varepsilon\left(t_{1}^{*}\left|\mathbf{e}_{1}\right|^{2}+\left(2\left|\mathbf{e}_{2}\right|^{2}+2\left|\mathbf{e}_{3}\right|^{2}\right)\left(1-t_{1}^{*}\right)\right.} \\
& =\frac{4|K|^{2}}{3} \frac{t_{1}^{*}\left(1-t_{1}^{*}\right)}{\varepsilon\left(t_{1}^{*}\left|\mathbf{e}_{1}\right|^{2}+\left(\left|\mathbf{e}_{1}\right|^{2}+\left|\mathbf{e}_{2}-\mathbf{e}_{3}\right|^{2}\right)\left(1-t_{1}^{*}\right)\right.} \\
& =\frac{4|K|^{2}}{3} \frac{t_{1}^{*}\left(1-t_{1}^{*}\right)}{\left(\varepsilon\left|\mathbf{e}_{1}\right|^{2}+\varepsilon\left(1-t_{1}^{*}\right)\left(\left|\mathbf{e}_{2}-\mathbf{e}_{3}\right|^{2}\right)\right.} .
\end{aligned}
$$

On the other hand, from (4.13), we know that the value of $t_{1}^{*}$ is given by

$$
t_{1}^{*}=1+\frac{\varepsilon\left|\mathbf{e}_{1}\right|^{2}}{\varepsilon\left|\mathbf{e}_{2}-\mathbf{e}_{3}\right|^{2}+\frac{4}{3}|K|\left(\beta, \nu_{2}\right)},
$$


from which, we deduce that

$$
\varepsilon\left|\mathbf{e}_{1}\right|^{2}+\varepsilon\left(1-t_{1}^{*}\right)\left|\mathbf{e}_{2}-\mathbf{e}_{3}\right|^{2}=-\frac{4}{3}\left(1-t_{1}^{*}\right)|K| \beta \cdot \nu_{2} .
$$

Now we substitute (4.41) into (4.39) and get

$$
\alpha(P)=\frac{|K| t_{1}^{*}}{-\beta \cdot \nu_{2}}
$$

since $t_{1}^{*} \neq 1$. Noting that $|K|=\frac{1}{2}\left|\mathbf{e}_{1}\right| \mathbf{n}_{1} \cdot \mathbf{m}$ with $\mathbf{m}=\mathbf{M}-\mathbf{V}_{1}$, the representation of $\alpha(P)$ becomes

$$
\alpha(P)=\frac{\left(\mathbf{M}-\mathbf{V}_{1}\right) t_{1}^{*} \cdot \nu_{1}}{-2 \beta \cdot \nu_{2}}
$$

The result immediately follows since $t_{1}^{*} \geq 2 / 3$.

The next lemma is the counterpart of the previous one for Case 2.

Lemma 4.2. Assume that, in Case $2, \varepsilon \leq \varepsilon_{2}^{*}$. Then the function $b_{P}^{*}$, the solution of (4.3), satisfies the inequality

$$
C_{*, 2} h_{K} \leq b_{P}^{*}(P) \leq C_{2}^{*} h_{K},
$$

where $C_{2}^{*}$ and $C_{*, 2}$ depend only on $\beta$ and on the minimum angle in $K$.

Proof. We follow the lines of the proof in the previous lemma. Remember that we have one inflow boundary edge $e_{1}$ and a different representation for $t$ which is given by (4.21). With this value of $t$ and the representation of $\alpha(P)$ in (4.37), the value of the function $b_{P}^{*}$ at the point $P$ is given by

$$
\begin{aligned}
\alpha(P) & =\frac{4|K|^{2}}{3} \frac{1}{\varepsilon\left(\frac{\left|\mathbf{e}_{1}\right|^{2}}{1-t_{2}^{*}}+\frac{2\left|\mathbf{e}_{2}\right|^{2}}{t_{2}^{*}}+\frac{2\left|\mathbf{e}_{3}\right|^{2}}{t_{2}^{*}}\right)} \\
& =\frac{4|K|^{2}}{3} \frac{t_{2}^{*}\left(1-t_{2}^{*}\right)}{\left(\varepsilon\left|\mathbf{e}_{1}\right|^{2}+\varepsilon\left(1-t_{2}^{*}\right)\left|\mathbf{e}_{2}-\mathbf{e}_{3}\right|^{2}\right)},
\end{aligned}
$$

where we have used the identity (4.10). On the other hand, from (4.20), we know that the value of $t_{2}^{*}$ is given by

$$
t_{2}^{*}=\frac{\varepsilon\left(\left|\mathbf{e}_{2}\right|^{2}+\left|\mathbf{e}_{3}\right|^{2}\right)}{\varepsilon\left|\mathbf{e}_{2}-\mathbf{e}_{3}\right|^{2} / 2-|K|\left(\beta, \nu_{1}\right) / 3} .
$$

From (4.46), after some algebraic manipulations, we deduce that

$$
\varepsilon\left|\mathbf{e}_{1}\right|^{2}+\varepsilon\left(1-t_{2}^{*}\right)\left|\mathbf{e}_{2}-\mathbf{e}_{3}\right|^{2}=-\frac{2}{3} t_{2}^{*}|K| \beta \cdot \nu_{1} .
$$

Now substituting (4.47) into (4.45) and noting that $t_{2}^{*} \neq 0$, we have

$$
\alpha(P)=\frac{2|K|\left(1-t_{2}^{*}\right)}{-\beta \cdot \nu_{1}} .
$$


Recalling that $|K|=\frac{1}{2}\left|\mathbf{e}_{1}\right| \mathbf{n}_{1} \cdot \mathbf{m}$ with $\mathbf{m}=\mathbf{M}-\mathbf{V}_{1}$, the representation of $\alpha(P)$ becomes

$$
\alpha(P)=\frac{\left(\mathbf{M}-\mathbf{V}_{1}\right)\left(1-t_{2}^{*}\right) \cdot \nu_{1}}{-\beta \cdot \nu_{1}} .
$$

The inequality (4.44) immediately follows since $t_{2}^{*} \leq 1 / 3$.

Theorem 4.2. Assume that, for every $K \in \tau_{h}$, the position of the internal node $P$ is chosen according to (4.14) in Case 1 or (4.21) in Case 2. Then the conditions (4.33) and (4.34) hold true with constants independent of $h$.

Proof. For Case 1, this is a direct consequence of Lemma 4.1 together with Lemma 3, Propositions 1 and 2 from Ref. 2. For Case 2, we use Lemma 4.2 together with Lemma 3, Propositions 1 and 2 from Ref. 2.

\section{Numerical Results}

In this section, we will present some numerical experiments showing the stabilizing features of the method under consideration. We remark that, in our method, the subgrid node is chosen according to the criteria (4.2). We will solve the problem (2.1) with $f=0$ and a nonzero Dirichlet boundary condition on a computational domain $\Omega=[0,1] \times[0,1]$ described in Fig. 6 . We take a midrange value for $\varepsilon$; $\varepsilon=0.001 . \beta=\gamma /|\gamma|$.

Experiment 1. Here we present the plain Galerkin solution of the problem on a set of successively refined meshes. We first take a set of coarse meshes which are made of 200, 800 and 3200 uniform triangular elements. Then we augment each coarse mesh by adding a single subgrid node, whose location is determined

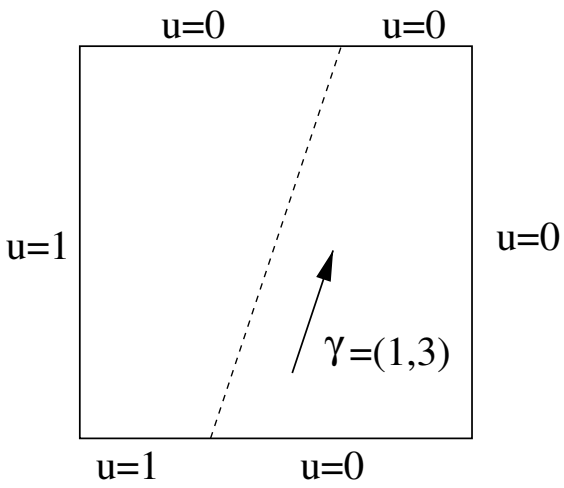

Fig. 6. Configuration of the test problem. 

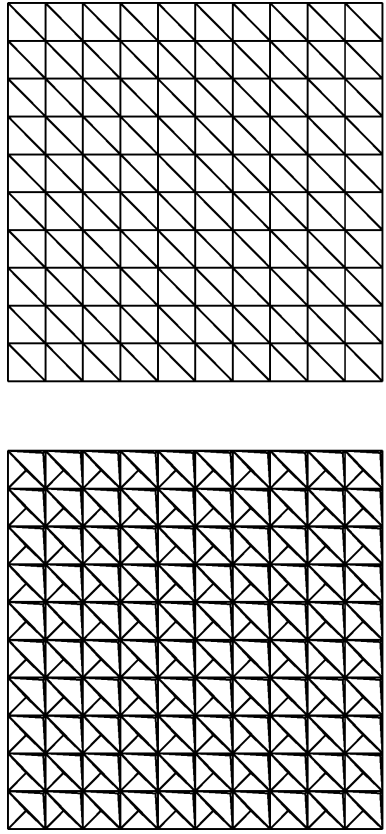
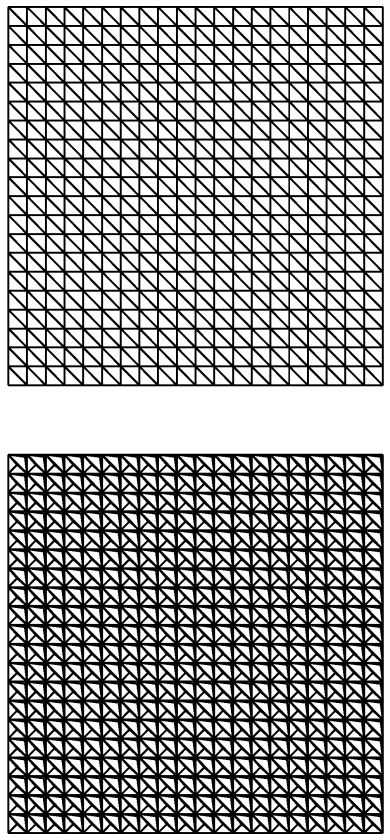
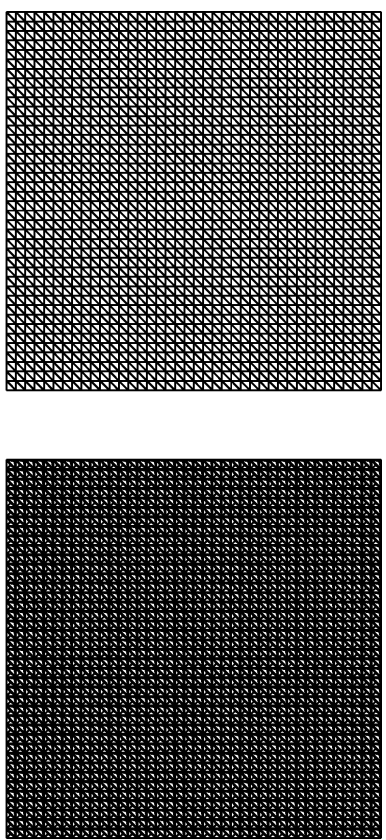

Fig. 7. Coarse meshes (above) and their corresponding augmented meshes (below).

according to (4.14) and (4.21) depending on the number of inflow edges, in each element and joining it to the coarse mesh points, thus forming three sub-triangles in each element of coarse mesh (see Fig. 7). The elevation plots of the complete solutions is displayed on the augmented meshes in the first row of Fig. 8. In the second row, the linear parts of the complete solutions are presented. We notice that both solutions are very close to each other. We further note that the test problem has an exact solution that exhibits an internal and a boundary layer. It is clear that our numerical solution is able to show the dominant characteristics of the exact solution at all levels of the mesh employed.

Experiment 2. In this experiment we compare the plain Galerkin solution on two classes of augmented meshes. We first take the set of coarse meshes in the previous experiment and augment these coarse meshes with two different choice of subgrid nodes. In the first choice, we take the subgrid node to be the barycentric coordinates of the element without taking the value of $\varepsilon$ into consideration. In the second choice, we locate the subgrid node according to (4.14) and (4.21) as in the previous experiment (Fig. 9). The elevation plots of the standard Galerkin solution on two choices of augmented meshes are shown in Fig. 10. We can conclude that the stabilizing effect of the method depends fairly on the enrichment of the grid by means of a suitably chosen subgrid. 


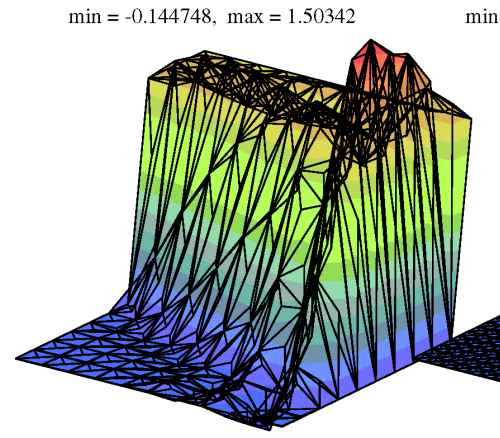

$\min =-0.0913032, \max =1.46848$

$\min =-0.0308674, \max =1.43528$

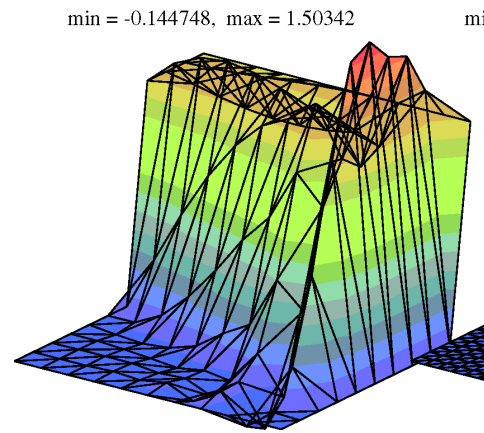

$\min =-0.0913032, \max =1.46848 \quad \min =-0.0308674, \max =1.43528$

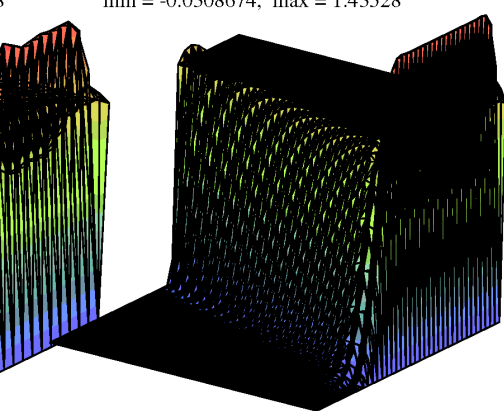

Fig. 8. Elevation plots of the complete solutions (above) and their linear parts (below).
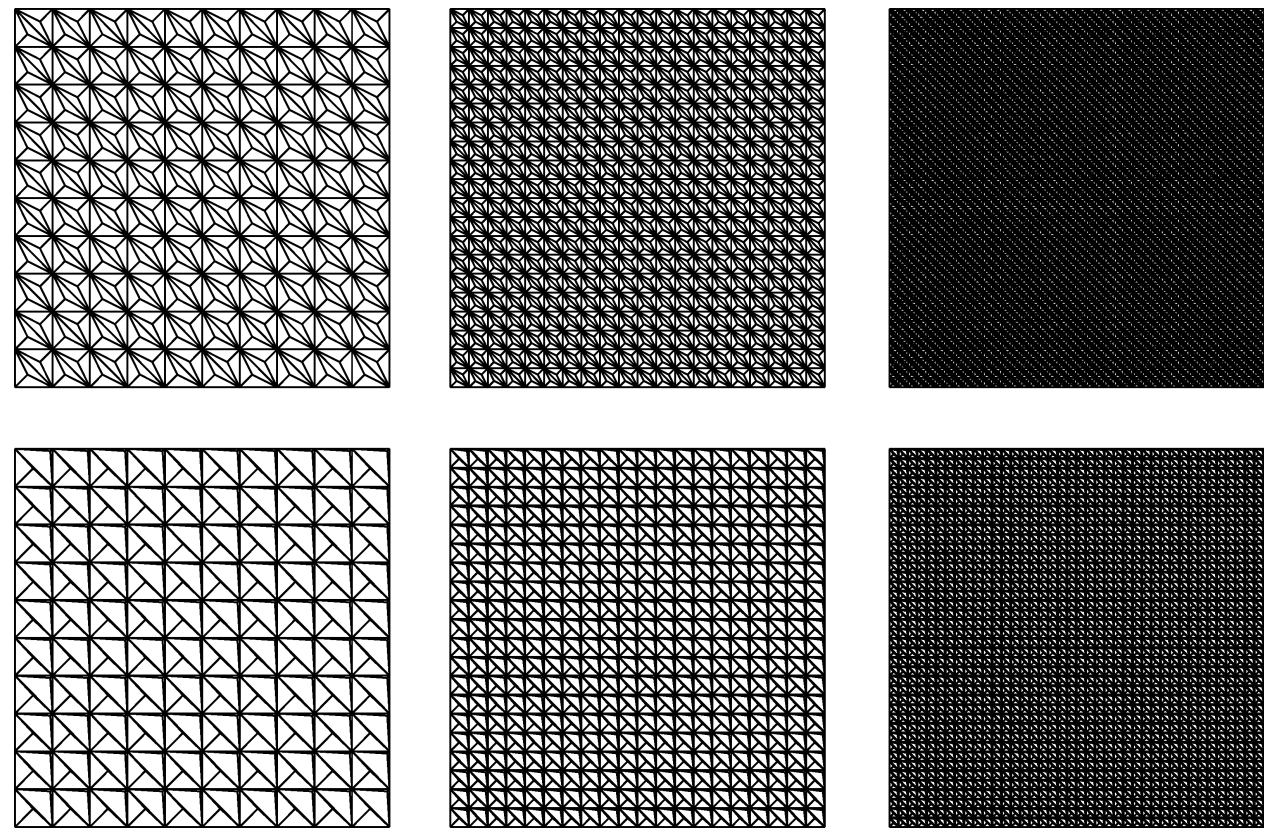

Fig. 9. Augmented meshes: Subgrid node; (i) located at the barycenter (upper row), (ii) located according to (4.14) or (4.21) (lower row). 


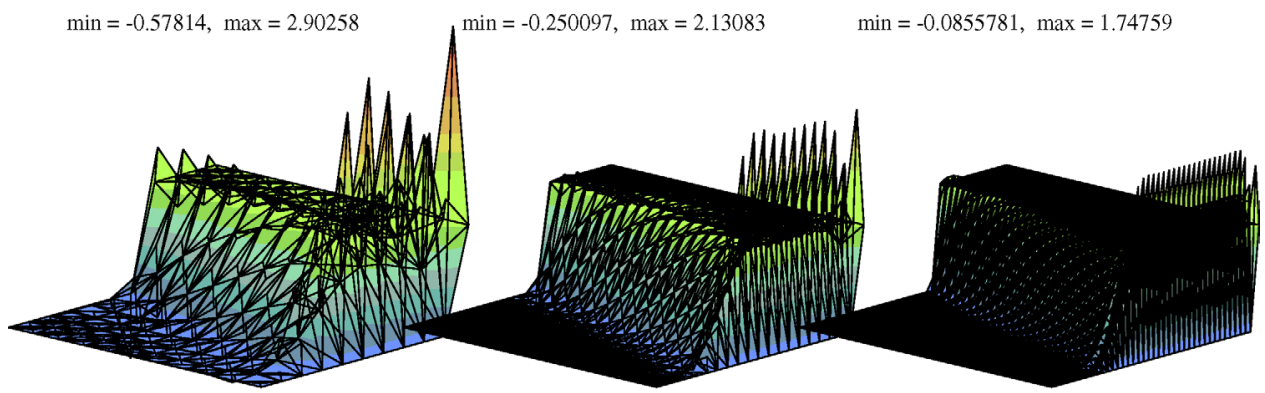

$\min =-0.144748, \max =1.50342$

$\min =-0.0913032, \max =1.46848$

$\min =-0.0308674, \max =1.43528$

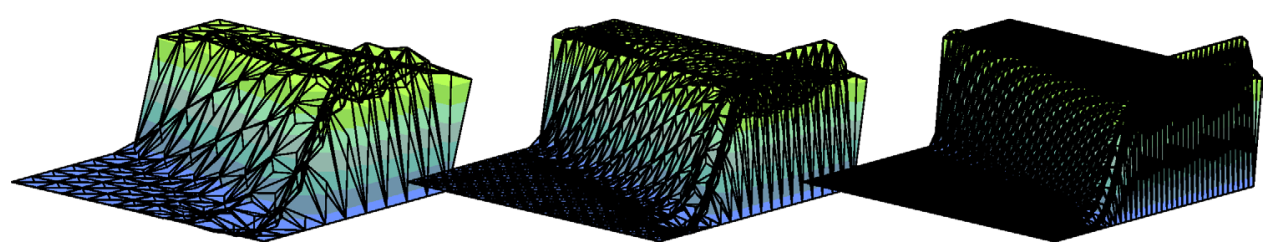

Fig. 10. Standard Galerkin solution on the augmented meshes in Fig. 9.

\section{Conclusion}

We considered the standard Galerkin Finite Element Method on a triangular mesh augmented with a suitably chosen subgrid for solving the convection-diffusion problem. We showed that our choice of subgrid is qualitatively the same as the subgrid suggested in a recent work of Brezzi and his coworkers. We further proved that the discrete solution on augmented grid satisfies the same a priori error estimates that are typically obtained with SUPG and RFB methods. Numerical experiments presented clearly confirms the theoretical findings.

\section{References}

1. F. Brezzi and D. Marini, Augmented spaces, two-level methods, and stabilizing subgrids, Int. J. Numer. Meth. Fluids 40 (2002) 31-46.

2. F. Brezzi, D. Marini and A. Russo, On the choice of a stabilizing subgrid for convection-diffusion problems, Comput. Meth. Appl. Mech. Engrg. 194 (2005) $127-148$.

3. F. Brezzi, D. Marini and A. Russo, Applications of the pseudo residual-free bubbles to the stabilization of convection-diffusion problems, Comput. Meth. Appl. Mech. Engrg. 166 (1998) 51-63.

4. F. Brezzi, T. J. R. Hughes, L. D. Marini, A. Russo and E. Suli, A priori error analysis of residual-free bubbles for advection-diffusion problems, SIAM J. Numer. Anal. 36 (1999) 1933-1948.

5. F. Brezzi, L. D. Marini and A. Russo, The residual-free bubbles for advection-diffusion problems: The general error analysis, Numer. Math. 85 (2000) 31-47. 
6. F. Brezzi, M. O. Bristeau, L. P. Franca, M. Mallet and G. Rogé, A relationship between stabilized finite element methods and the Galerkin method with bubble functions, Comput. Meth. Appl. Mech. Engrg. 96 (1992) 117-129.

7. F. Brezzi, G. Hauke, D. Marini and G. Sangalli, Link-cutting bubbles for the stabilization of convection-diffusion-reaction problems, Math. Mod. Meth. Appl. Sci. 13 (2003) 445-461.

8. F. Brezzi and A. Russo, Choosing bubbles for advection-diffusion problems, Math. Mod. Meth. Appl. Sci. 4 (1994) 571-587.

9. A. N. Brooks and T. J. R. Hughes, Streamline upwind/Petrov-Galerkin formulations for convection dominated flows with particular emphasis on the incompressible Navier-Stokes equations, Comput. Meth. Appl. Mech. Engrg. 32 (1982) 199-259.

10. L. P. Franca, S. L. Frey and T. J. R. Hughes, Stabilized finite element methods: I. Application to the advective-diffusive model, Comput. Meth. Appl. Mech. Engrg. 95 (1992) 253-276.

11. L. P. Franca and A. Nesliturk, On a two-level finite element method for the incompressible Navier-Stokes equations, Int. J. Numer. Meth. Engrg. 52 (2001) 433-453.

12. L. P. Franca, A. Nesliturk and M. Stynes, On the stability of residual-free bubbles for convection-diffusion problems and their approximation by a two-level finite element method, Comput. Meth. Appl. Mech. Engrg. 166 (1998) 35-49.

13. L. P. Franca and L. Tobiska, Stability of the residual free bubble method for bilinear finite elements on rectangular grids, IMA J. Numer. Anal. 22 (2002) 73-87.

14. T. J. R. Hughes, L. P. Franca and G. Hulbert, A new finite element formulation for computational fluid dynamics: VII. The Galerkin/least squares method for advective-diffusive equations, Comput. Meth. Appl. Mech. Engrg. 73 (1989) 173-189.

15. U. Risch, Convergence analysis of the residual free bubble method for bilinear elements, SIAM J. Numer. Anal. 39 (2001) 1366-1379.

16. H.-G. Roos, M. Stynes and L. Tobiska, Numerical Methods for Singularly Perturbed Differential Equations (Springer Verlag, 1996).

17. G. Sangalli, Global and local error analysis for the residual-free bubbles method applied to advection-dominated problems, SIAM J. Numer. Anal. 38 (2000) $1496-1522$. 